\title{
Hepatitis C virus-associated hepatocellular carcinoma as a second primary malignancy: exposing an overlooked presentation of liver
}

\section{cancer}

This article was published in the following Dove Press journal:

Journal of Hepatocellular Carcinoma

\section{Dima Dandachi,, ${ }^{1,2}$ Manal Hassan, ${ }^{3}$ Ahmed Kaseb, ${ }^{4}$ Georgios Angelidakis, ${ }^{2}$ Harrys A Torres ${ }^{2,4}$ \\ 'Department of Infectious Diseases, Baylor College of Medicine, Houston, TX, USA; ${ }^{2}$ Department of Infectious Diseases, Infection Control and Employee Health, The University of Texas MD Anderson Cancer Center, Houston, TX, USA; ${ }^{3}$ Department of Epidemiology, The University of Texas Medical School, Houston, TX, USA; ${ }^{4}$ Department of Gastroenterology, Hepatology, and Nutrition, The University of Texas MD Anderson Cancer Center,} Houston, TX, USA

\section{Video abstract}

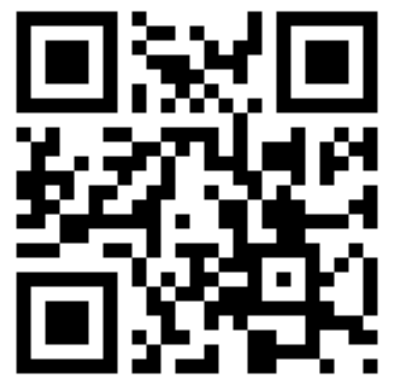

Point your SmartPhone at the code above. If you have a $Q R$ code reader the video abstract will appear. Or use: http://youtu.be/_T-XM-MwNUs

Correspondence: Harrys A Torres Department of Infectious Diseases, Infection Control and Employee Health, Unit 1460, The University of Texas MD Anderson Cancer Center, 1515 Holcombe Blvd, Houston, TX, 77030, USA

Tel +I 7137926830

Fax +17137456839

Email htorres@mdanderson.org
Introduction: Chronic hepatitis $\mathrm{C}$ virus (HCV) infection is one of the leading causes of hepatocellular carcinoma (HCC) worldwide. Antiviral therapy in patients with HCV infection reduces the risk of primary $\mathrm{HCC}$ development by $71 \%-75 \%$. HCV-infected patients with different primary cancers are also at risk for HCC development as a second primary malignancy (HCC-SPM). Limited information is available on the occurrence and characteristics of HCCSPM. Herein, we determine the prevalence and clinical features of HCV-associated HCC-SPM when compared to primary HCC.

Materials and methods: Patients with HCV-associated HCC seen at MD Anderson Cancer Center (2011-2017) were enrolled in a prospective observational study. Patients with multiple cancers diagnosed simultaneously or with hepatitis B virus or HIV coinfections were excluded. At enrollment, patients completed a questionnaire on medical history and HCC risk factors. Information on demographics, comorbidities, HCV treatment, tumor characteristics, treatment modalities, and virologic and oncologic outcomes were extracted from the medical records.

Results: Among 171 consecutive patients with HCV-associated HCC enrolled, 26 (15\%) had HCC-SPM. Most of the underlying primary cancers were solid tumors (85\%). In $12(46 \%)$ of these patients, the diagnosis was made incidentally while undergoing surveillance for primary malignancies, and the majority ( $81 \%$ ) had their primary cancer in remission. Most patients $(72 \%)$ with documented HCV viral load had chronic viremia due to lack of diagnosis, lack of treatment, or prior unsuccessful treatment of HCV infection and only $28 \%$ had undetectable viral load following successful antiviral therapy. The overall median survival for both groups was 29 months (95\% CI: 23-35) without difference between groups $(p=0.2)$.

Conclusion: Cancer patients with any malignancies must be screened for HCV as HCCSPM can develop in $15 \%$ of infected patients. Early HCV diagnosis and treatment should be attempted to prevent the development of HCC-SPM, a condition associated with high mortality in cancer survivors.

Keywords: liver cancer, cancer prevention, cancer survivors, DAA, HCV treatment

\section{Introduction}

Chronic hepatitis $\mathrm{C}$ virus ( $\mathrm{HCV}$ ) infection is a leading cause of hepatocellular carcinoma (HCC) worldwide. Relative to the uninfected population, patients with HCV infection have an $\sim 10$ - to 20 -fold greater risk of HCC. ${ }^{1}$ Antiviral therapy for chronic HCV infection reduces the risk of primary HCC (HCC-P) development by $71 \%-75 \%$. $^{2,3}$ With an increasing number of cancer survivors and aging patient population, the number of 
patients with second primary malignancies (SPMs) is also increasing. HCV-infected patients with different primary cancers are at risk for HCC as an SPM (HCC-SPM). ${ }^{4}$

In a retrospective analysis conducted by our group with 642 patients seen between 2008 and 2011 with chronic HCV infection and any type of primary cancer, HCC-SPMs developed in $7 \%$ of them. ${ }^{5}$ In the first study to evaluate the predictors of $\mathrm{HCC}$ as an SPM in HCV-infected patients, we found that patients with HCC-SPMs were more likely to have solid tumors and progressive malignancy. The most common solid tumors were basal cell carcinoma (21\%), prostate cancer $(14 \%)$, and thyroid cancer $(14 \%)$. Most common treatment modalities were surgery $(69 \%)$, followed by chemotherapy (34\%), most commonly with monoclonal antibodies (rituximab, bevacizumab, and trastuzumab). In addition, patients with HCC-SPMs were less likely to achieve sustained virologic response compared to patients in whom HCC did not develop. ${ }^{6}$

In the study described herein, we prospectively analyzed the prevalence and clinical features of HCV-associated HCCSPMs compared with HCC-P.

\section{Materials and methods}

\section{Study design and patient population}

Patients with HCV-associated HCC seen at The University of Texas MD Anderson Cancer Center from 2011 to 2017 were enrolled in a prospective observational study at the time of HCC diagnosis. HCC was diagnosed by evaluation of liver mass by abdominal imaging (ultrasound or computerized tomography scan) and/or biopsy. Patients with multiple cancers diagnosed simultaneously or with hepatitis B virus or HIV coinfections were excluded. HCV diagnosis was made based on self-reported medical history of $\mathrm{HCV}$ infection and/or treatment or current active infection based on HCV RNA level.

At enrollment, patients completed a questionnaire on their medical history and HCC risk factors. The HCC risk factors investigated included tobacco and alcohol abuse, obesity, diabetes mellitus, and chronic liver diseases. The following information was extracted from the medical records: demographics (age, sex, race/ethnicity), comorbidities, prior history of malignancy, laboratories values at baseline, tumor characteristics, staging and treatment modalities for HCC, death, and overall survival at 5 years. Available data on $\mathrm{HCV}$ infection (past or active infection), HCV genotype, and antiviral therapy at the time of HCC diagnosis was also recorded.

HCC was defined as SPM if patients did have a prior history of cancer before the HCC diagnosis. Variables com- pared between patients with HCC-SPM and HCC-P include demographics, risk factors for HCC, clinical presentation and therapeutic approaches for HCC, and virologic and oncologic outcome.

\section{Ethics/institutional review board approval of research}

The study protocol was approved by The MD Anderson Institutional Review Board. A written informed consent was obtained from patients. The procedures followed were in accordance with the ethical standards.

\section{Statistical analysis}

To compare the demographic and clinical characteristics of the HCC-SPM and HCC-P patients, descriptive analysis was used to calculate frequencies, a Pearson $\chi^{2}$ test was used for categorical variables, and an independent Student $t$-test was used for continuous variables. $P$-values were used to assess for statistical significance, with $p$-values $<0.05$ considered to indicate significance. All statistical tests were conducted using SPSS version 24 (IBM Corporation, Armonk, NY, USA).

\section{Results}

We identified 191 patients with HCV-associated HCC. Twenty patients had either hepatitis B virus or HIV coinfection, or had multiple cancers diagnosed simultaneously and were excluded. We included 171 consecutive patients in the final analysis. Most of them were more than 50 years old $(97 \%)$, white $(65 \%)$, and male (78\%). Twenty-six (15\%) patients had $\mathrm{HCV}$-associated HCC-SPMs. Most of the underlying primary cancers were solid tumors (85\%), predominantly skin (31\%), breast (15\%), and lung (12\%) cancers (Figure 1). The most common treatment modalities for the first primary cancers were surgery (78\%) and chemotherapy (50\%) (Table 1).

At the time of diagnosis, $99 \%$ of the patients had liver cirrhosis. Twelve patients (46\%) were asymptomatic and had HCC-SPMs incidentally diagnosed while undergoing surveillance for primary malignancies. When we compared HCC-SPMs and HCC-P, we found no differences between the 2 patient groups in smoking history, alcohol intake, body mass index, diabetes mellitus history, family history of liver cancer, or HCC pathology. Patients with HCC-SPMs had better liver function test results and tumor characteristics (Table 2), but we could not determine the adherence to routine medical care in the 2 groups. The majority of patients $(50 \%)$ with $\mathrm{HCC}$ were treated with transarterial chemoembolization and transarterial radioembolization with yttrium- $90,7 \%$ had 
Primary cancers

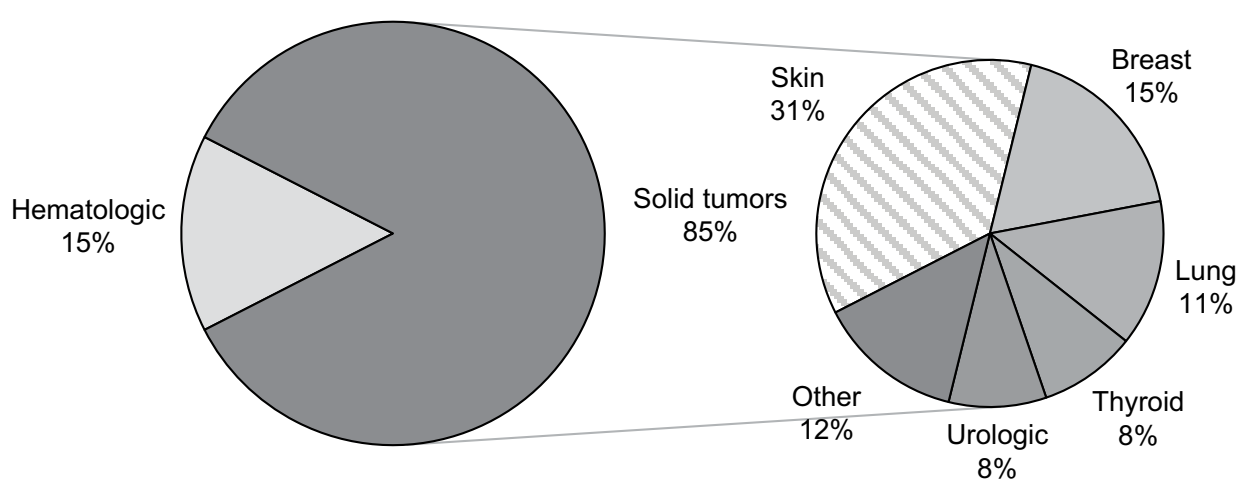

Figure I Distribution of primary malignancies.

Table I Comparison of baseline clinical characteristics in the HCC-SPM and HCC-P patient groups $(n=I 7 I)$

\begin{tabular}{|c|c|c|c|}
\hline Characteristics & HCC-SPM $(n=26)$ & HCC-P $(n=145)$ & $p$-value \\
\hline \multicolumn{4}{|l|}{ Demographics } \\
\hline Median age, years (range) & $64(50-79)$ & $60(39-85)$ & 0.35 \\
\hline Male sex & $18(69)$ & $115(79)$ & 0.25 \\
\hline White race & $18(69)$ & $93(64)$ & 0.78 \\
\hline \multicolumn{4}{|l|}{ Past medical history } \\
\hline History of smoking (current or past) & $20(77)$ & $100(69)$ & 0.74 \\
\hline Alcohol intake (current or past) & $2 I(8 I)$ & I I4 (79) & 0.53 \\
\hline Median baseline BMI & 26 & 28 & 0.35 \\
\hline History of DM & $3(12)$ & $27(19)$ & 0.41 \\
\hline Presence of hepatic steatosis & I (4) & $18(12)$ & 0.31 \\
\hline \multicolumn{4}{|l|}{ Laboratory values at baseline } \\
\hline Median $\alpha$-fetoprotein & 19 & 35 & 0.54 \\
\hline Median lactate dehydrogenase level (IU/L) & 537 & 603 & 0.02 \\
\hline Median ALT level (IU/L) & 59 & 76 & 0.18 \\
\hline Median alkaline phosphatase level (IU/L) & 109 & 159 & $<0.01$ \\
\hline Median INR & 0.9 & 1.2 & 0.08 \\
\hline Median total bilirubin level (mg/dL) & 0.9 & 1.2 & 0.13 \\
\hline Median serum albumin level (g/dL) & 4 & 3.8 & 0.11 \\
\hline
\end{tabular}

Note: Data are presented as $\mathrm{n}(\%)$ unless otherwise specified.

Abbreviations: ALT, alanine aminotransferase; BMI, body mass index; DM, diabetes mellitus; HCC-P, hepatocellular carcinoma as primary; HCC-SPM, hepatocellular carcinoma as second primary malignancy; INR, international normalized ratio.

radiofrequency ablation, cryoablation, or ablation by ethanol, and $6 \%$ had surgical resection or transplant. However, $27 \%$ of the patients presented with advanced disease and received sorafenib systemic therapy and $8 \%$ received palliative supportive care. The difference in therapeutic approaches used for the treatment of HCC was not statistically significant $(p=0.16)$ (Table 2).

At the time of HCC diagnosis, most patients (72\%) who had either HCC-SPMs or HCC-P with documented HCV viral load $(n=88)$ had chronic viremia due to lack of diagnosis, lack of treatment, or prior unsuccessful treatment of HCV infection and only $28 \%$ had undetectable viral load following successful antiviral treatment. Genotype 1 was the predominant HCV genotype (70\%), followed by genotype $2(14 \%)$ ). A total of 97 patients $(57 \%)$ were treated for $\mathrm{HCV}$ infection. Those given direct-acting antivirals had a significantly higher sustained virologic response rate than did those who were given interferon-based therapy $(81 \%$ vs $41 \% ; p<0.01$ )

Among the 26 patients with HCC-SPMs, the majority (81\%) had their primary cancer in remission, $11 \%$ had stable disease under surveillance, and $8 \%$ had progression or relapse 
Table 2 Comparison of HCC-SPM and HCC-P baseline pathology, staging, and treatment $(n=I 7 I)$

\begin{tabular}{|c|c|c|c|}
\hline Characteristics & HCC-SPM $(n=26)$ & HCC-P $(n=145)$ & p-value \\
\hline HCC pathology & & & 0.40 \\
\hline Well-moderately differentiated & $5(19)$ & $13(9)$ & \\
\hline Moderately differentiated & $10(38)$ & $32(22)$ & \\
\hline Moderately-poorly differentiated & I (4) & $12(8)$ & \\
\hline \multicolumn{4}{|l|}{$\mathrm{HCC}$ characteristics and staging } \\
\hline Median tumor size $(\mathrm{cm})$ & 3.4 & 5.4 & 0.01 \\
\hline \multicolumn{4}{|l|}{ Number of nodules } \\
\hline Solitary nodule & 14 (54\%) & $48(33 \%)$ & 0.06 \\
\hline Multinodular & $12(46 \%)$ & $91(63 \%)$ & \\
\hline Presence of vascular thrombosis & $5(19)$ & $54(37)$ & 0.14 \\
\hline Presence of metastasis & $2(8)$ & $30(2 I)$ & 0.10 \\
\hline Child-Pugh classification & & & 0.23 \\
\hline$A$ & $19(73)$ & $85(59)$ & \\
\hline B & $5(19)$ & $50(34)$ & \\
\hline C & $2(8)$ & $8(6)$ & \\
\hline TNM classification & & & 0.04 \\
\hline Stage-IA/IB & $9(35 \%)$ & $19(13 \%)$ & \\
\hline Stage II & $5(19 \%)$ & $16(11 \%)$ & \\
\hline Stage-IIIA/IIIB & $2(8 \%)$ & $30(21 \%)$ & \\
\hline Stage-IVA/IVB & $10(38 \%)$ & $76(55 \%)$ & \\
\hline $\mathrm{HCC}$ treatment & & & 0.16 \\
\hline Surgical resection or transplant & $4(16 \%)$ & $7(5 \%)$ & \\
\hline Local therapy (TACE, TARE) & II (44\%) & $75(53 \%)$ & \\
\hline Ablation therapy (RFA, cryoablation, ETOH ablation) & $3(12 \%)$ & $9(6 \%)$ & \\
\hline Sorafenib systemic therapy & $5(20 \%)$ & $42(29 \%)$ & \\
\hline Supportive care & $2(8 \%)$ & $10(7 \%)$ & \\
\hline
\end{tabular}

Note: Data are presented as $\mathrm{n}(\%)$ unless otherwise specified.

Abbreviations: ETOH, ethanol; HCC, hepatocellular carcinoma; HCC-SPM, hepatocellular carcinoma as second primary malignancy; RFA, radiofrequency ablation; TACE, transarterial chemoembolization; TARE, transarterial radioembolization with yttrium-90. HCC-P, hepatocellular carcinoma as primary.

at the time of HCC diagnosis. The overall median survival for both groups was 29 months (95\% CI: 23-35) without statistically significant differences in the survival between groups (Figure 2).

\section{Discussion}

To our knowledge, this is the first prospective analysis of the occurrence and characteristics of HCV-associated HCCSPMs. In this prospective study, we found that chronic $\mathrm{HCV}$ infection can lead to HCC-SPMs in up to $15 \%$ of patients. This study is a validation of the findings reported in a retrospective series from our group, but with a higher rate of HCC-SPM occurrence as compared to $7 \%$ from our previous report, ${ }^{6}$ likely due to better study design.

Many factors affect the natural progression of $\mathrm{HCV}$ infection. It is difficult to determine exactly the mechanism leading to the association between HCV and high incidence of hepatic and extrahepatic malignancies. ${ }^{4}$ At present, most cancer centers do not screen patients for $\mathrm{HCV}$ routinely. ${ }^{7}$ $\mathrm{HCV}$ testing in cancer patients is vital to identify chronically infected patients with any cancer. ${ }^{4}$ The development of HCC in our patients potentially would have been very low with more effective screening and treatment of $\mathrm{HCV}$ infection, extrapolated from a significant reduction in the risk of HCC-P development following $\mathrm{HCV}$ eradication. ${ }^{2}$

Baseline characteristics and clinical outcomes did not differ significantly between the HCC-SPM and HCC-P groups. Patients with HCC-SPMs had considerably smaller tumors and better liver function test results. HCC-P group of patients also had more advanced TNM staging at presentation probably related to earlier diagnosis and better linkage to care in patients undergoing routine cancer surveillance for their primary malignancies.

Our study has several limitations. First, it was a singlecenter study. Second, our patients were enrolled prospectively at the time of HCC diagnosis. Some of the details regarding $\mathrm{HCV}$ treatment and cancer treatment (eg, chemotherapy, steroids) of their non-HCC primary cancer were not available, which could have an impact on viral replication, HCC development, and progression. ${ }^{8}$ Third, some patients completed their HCC treatment outside MD Anderson, which may have affected the outcomes. 


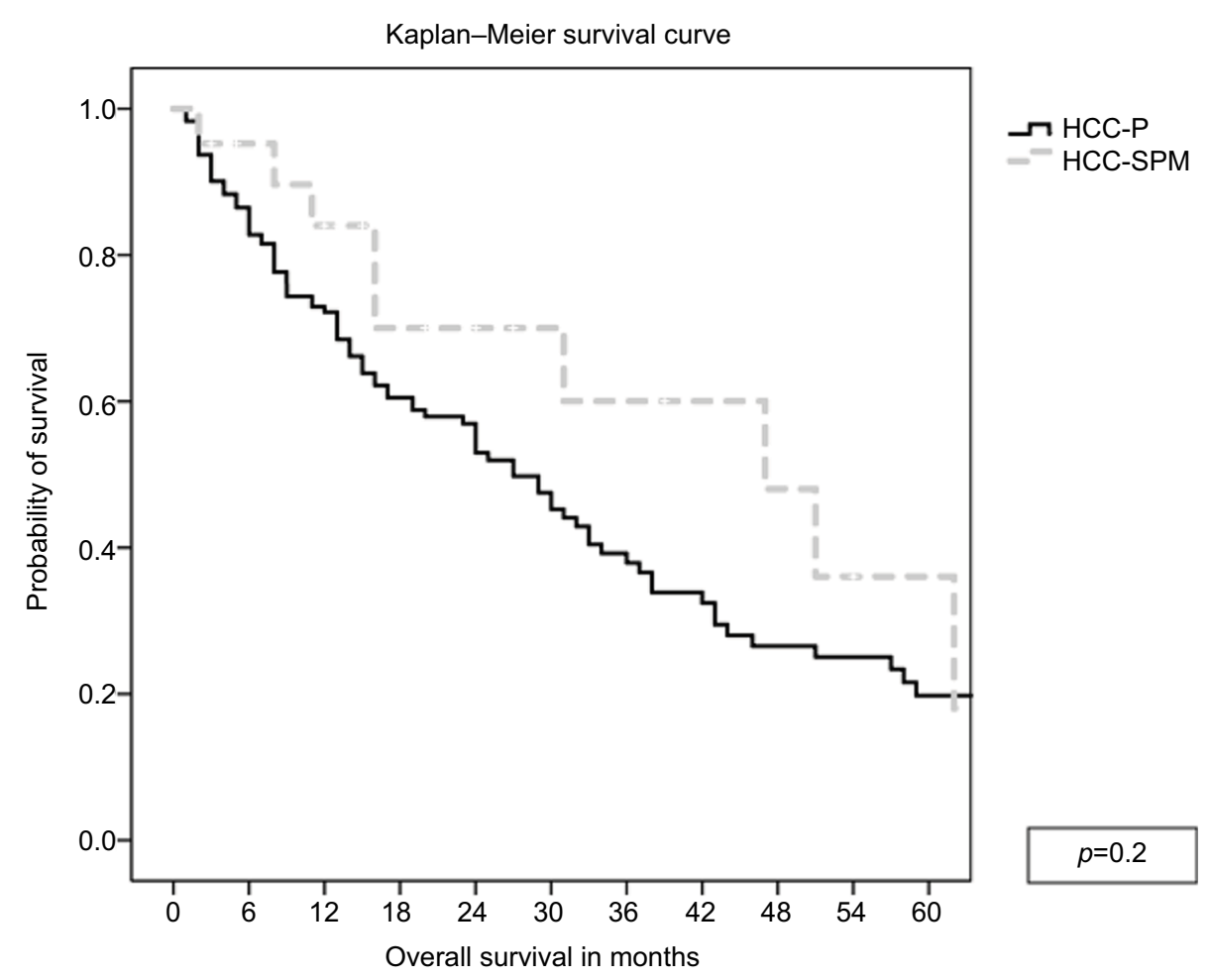

Figure 2 Survival curve analysis comparing patients with chronic HCV infection and HCC-SPM or HCC-P.

Abbreviations: HCC-P, hepatocellular carcinoma as primary; HCC-SPM, hepatocellular carcinoma as second primary malignancy; HCV, hepatitis C virus.

\section{Conclusion}

As increased awareness of the occurrence of second primary $\mathrm{HCC}$ in HCV-infected cancer patients, a not uncommon but lethal condition, is needed. Early HCV infection diagnosis paired with prompt treatment is required to prevent the development of HCC-SPMs, a condition associated with high mortality in cancer survivors.

\section{Acknowledgment}

The authors thank Mr Donald Norwood of the Department of Scientific Publications at MD Anderson Cancer Center for editorial assistance. This study was presented in part at The International Liver Congress, April 11-15, 2018, Paris, France. The poster's abstract was published in Hepatology, 2018 (68): S365-604 as a poster presentation. DOI: $\underline{\mathrm{https}: / /}$ doi.org/10.1016/S0168-8278(18)31114-0.

\section{Disclosure}

The authors report no conflicts of interest in this work.

\section{References}

1. Massarweh NN, El-Serag HB. Epidemiology of hepatocellular carcinoma and intrahepatic cholangiocarcinoma. Cancer Control. 2017;24(3):1073274817729245.

2. Ioannou GN, Green PK, Berry K. HCV eradication induced by directacting antiviral agents reduces the risk of hepatocellular carcinoma. J Hepatol. Epub 2017 Sept 5.

3. Ryerson AB, Eheman CR, Altekruse SF, et al. Annual Report to the Nation on the Status of Cancer, 1975-2012, featuring the increasing incidence of liver cancer. Cancer. 2016;122(9):1312-1337.

4. Torres HA, Shigle TL, Hammoudi N, et al. The oncologic burden of hepatitis C virus infection: a clinical perspective. CA Cancer J Clin. 2017;67(5): 411-431.

5. Torres HA, Mahale P, Blechacz B, et al. Effect of hepatitis $\mathrm{C}$ virus infection in patients with cancer: addressing a neglected population. $J$ Natl Compr Canc Netw. 2015;13(1):41-50.

6. Mahale P, Kaseb AO, Hassan MM, Torres HA. Hepatocellular carcinoma as a second primary cancer in patients with chronic hepatitis $\mathrm{C}$ virus infection. Dig Liver Dis. 2015;47(4):348-349.

7. Angelidakis G, Hwang JP, Dandachi D, et al. Universal screening for hepatitis C: a needed approach in patients with hematologic malignancies. J Viral Hepat. Epub 2018 Apr 16.

8. Torres HA, Hosry J, Mahale P, Economides MP, Jiang Y, Lok AS. Hepatitis $\mathrm{C}$ virus reactivation in patients receiving cancer treatment: a prospective observational study. Hepatology. 2018;67(1):36-47. 


\section{Publish your work in this journal}

The Journal of Hepatocellular Carcinoma is an international, peerreviewed, open access journal that offers a platform for the dissemination and study of clinical, translational and basic research findings in this rapidly developing field. Development in areas including, but not limited to, epidemiology, vaccination, hepatitis therapy, pathology and molecular tumor classification and prognostication are all considered for publication. The manuscript management system is completely online and includes a very quick and fair peer-review system, which is all easy to use. Visit http://www.dovepress.com/testimonials.php to read real quotes from published authors.

Submit your manuscript here: https://www.dovepress.com/journal-of-hepatocellular-carcinoma-journal 